\title{
The warped Spindle NGC 2685
}

\author{
Gyula Józsa* \\ RAIUB, Bonn, Germany \\ E-mail: gjozsalastro.uni-bonn.de

\section{Tom Oosterloo} \\ ASTRON, Dwingeloo, The Netherlands \\ E-mail: oosterloodastron.nl

\section{Uli Klein} \\ RAIUB, Bonn, Germany \\ E-mail: ukleineastro.uni-bonn.de
}

\begin{abstract}
We present HI- and optical (i'-band) data of the well known "Spindle" galaxy NGC 2685, along with our tilted-ring analysis of the obtained HI data cubes. The optical appearance of the galaxy on the sky suggests that this galaxy is a classical polar-ring galaxy with an additional outer HI-ring perpendicular to the inner one. Our observations show that this is not the case. While it is true that the orbits of the gas change their orientations substantially towards larger radii, the HI does not form two distinct rings but rather a coherent structure, an extreme warp with an extremely high degree of symmetry. The optical appearance of NGC 2685 resembles the total HI intensity to great detail so that it is likely that an extreme warp is also present in an extended stellar disk.
\end{abstract}

Baryons in Dark Matter Halos

5-9 October 2004

cat /dev Novigrad, Croatia

* Speaker. 


\section{Introduction}

The famous "Spindle"-galaxy NGC 2685 got its name because of the dust-lanes that cross an inner stellar body of a spectroscopically confirmed S0 galaxy. They belong to a rotationally supported structure containing gas and stars, following nearly polar orbits with respect to the central stellar body at small radii. NGC 2685 was therefore classified as a polar-ring galaxy (Whitmore et al. 1990). Furthermore, NGC 2685 shows an outer HI component, also visible in the stellar light, that lies perpendicular to the "polar ring" and seems to be aligned with the S0 central body of the galaxy.

In this contribution we present studies of the kinematics and the morphology of this galaxy, the structure and formation history of which is still heavily discussed (e.g. Schinnerer \& Scoville 2002 and citations therein).

\section{Observations and data analysis}

HI data cubes were obtained from $4 \times 12 \mathrm{~h}$ observations with the WSRT which were reduced using the Miriad reduction software package, as well as optical (i'-band) images resulting from an (13350 s on-source) observation with the INT and subsequent data reduction using the GaBoDS WFC reduction pipeline, established by Schirmer et al. in 2003 (HI: $\sigma_{\mathrm{rms}} \gtrsim 3 \cdot 10^{18}$ atoms cm ${ }^{-2}$, i': $\sigma_{\text {rms }} \gtrsim 25.8 \mathrm{mag} \operatorname{arcsec}^{-2}$ ). We used a $\chi^{2}$-minimisation method to fit a tilted-ring model (see poster contribution of Kenn et al. and talk of Józsa et al., this conference) to the obtained HI data cubes in order to parametrise the morphology and the kinematics of the HI component.

\section{Results}

The optical appearance of the stellar component of NGC 2685 resembles that of the HI in a great detail, at least at large radii, suggesting that both belong to the same structure.

It is evident from an inspection of the HI data cubes alone that the HI component of the galaxy forms a morphologically and kinematically coherent structure with a high degree of symmetry at all radii. This means that the HI component of NGC 2685 lies in one heavily warped disk.

Our analysis shows that the rotation velocity increases at larger radii, revealing a change of the gravitational potential with the orientation of the disk. This implies that the overall potential of the galaxy is not spherically symmetric.

The data resulting from our observations resemble the appearance of a galactic disk that forms from a single accretion event under the influence of a triaxial figure-rotating halo, as has been discussed by Peletier \& Christadoulou (1992) in a case study of NGC 2685.

\section{References}

[1] Peletier, R. F., Christadoulou, D. M., 1993, AJ 105, 1378

[2] Schinnerer, E., Scoville, N., 2002, ApJ, 577, 103

[3] Whitmore, B. C., Lucas, R. A., McElroy, D. B., Steiman-Cameron, T. Y., Sackett, P. D., Olling, R. P., 1990, AJ, 100, 1489 
a)


Figure 1: a) Multicolour image (B, V, and R band) of NGC 2685 (Image courtesy of the Isaac Newton Group of Telescopes, La Palma). b) Parameters of our best-fi t model, from top to bottom: HI surface-density, warp angle, position angle, inclination, and rotation velocity. As the warp in the galaxy starts at the centre, the warp angle is calculated with respect to the outer disk.

a)



d)

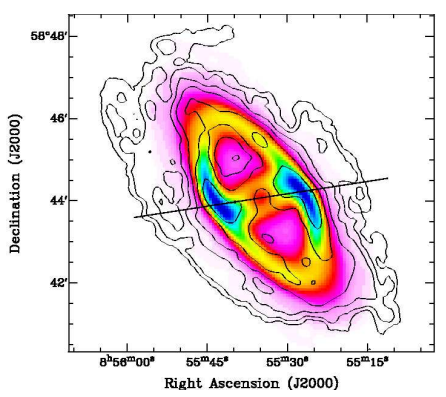

b)
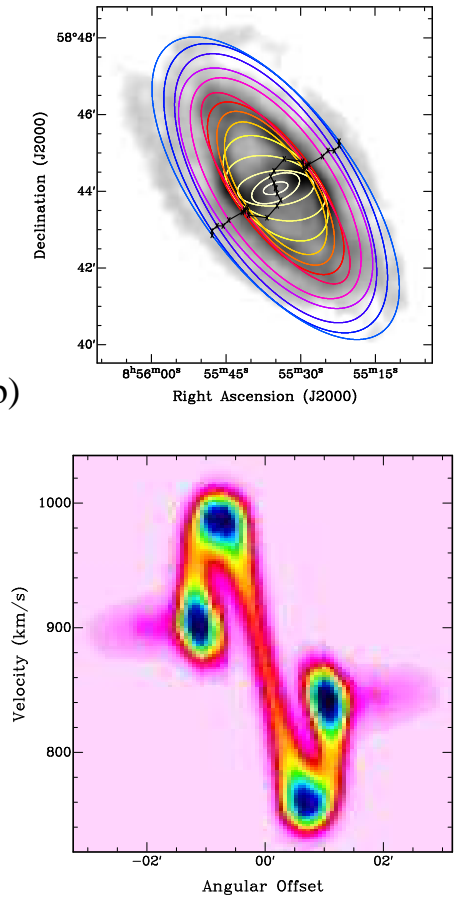

c)
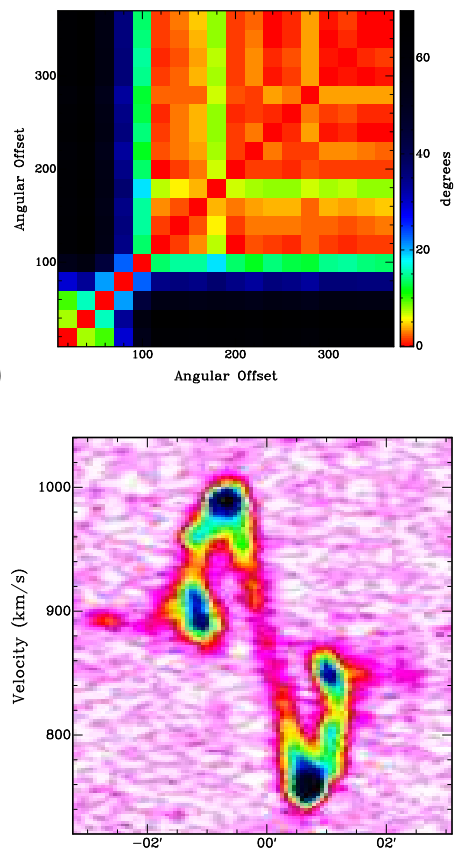

Angular Offset

Figure 2: Our observations and illustrations of our best-fit model: a) An overlay of the HI total intensity map (contours: $0.6,1.2,2.4,4.8,9.6 \mathrm{M}_{\odot} / \mathrm{pc}^{2}$ ) on the optical i' band image (greyscale). b) An overlay of a projection of fitted rings (coloured ellipses) on the HI total intensity map (greyscale) along with the line-of-nodes from our model (solid line). c) A "Tiltogram" showing the tilt angle of the modelled disk with itself at two radii. The axis units are arcsec. d) An overlay of the HI total intensity map (contours: $0.3,0.6,1.2,2.4,4.8,9.6 \mathrm{M}_{\odot} / \mathrm{pc}^{2}$ ) of our observations on the total intensity maps from our model. The solid line marks the direction along which the position-velocity diagrams e) and f) and those in Fig. 3 are carried out. e) Position-velocity diagram of the model carried out along the solid line in d). f) Position-velocity diagram of our observations carried out along the solid line in d). 
a)

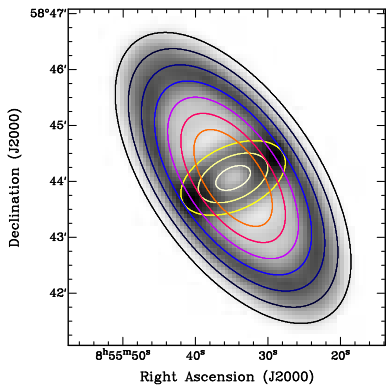

d)

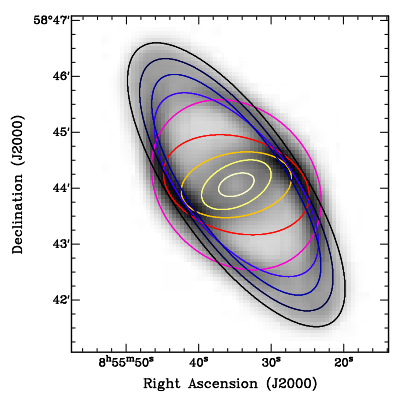

b)

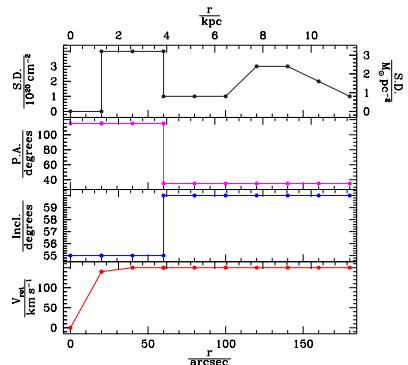

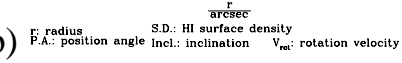

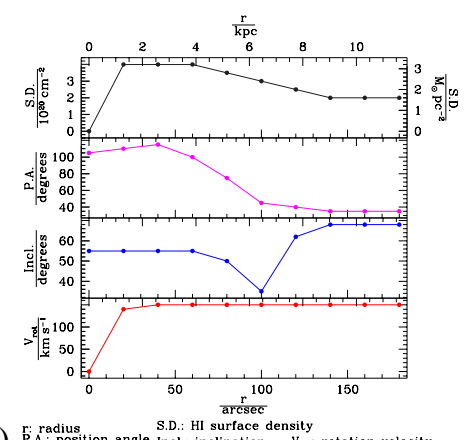



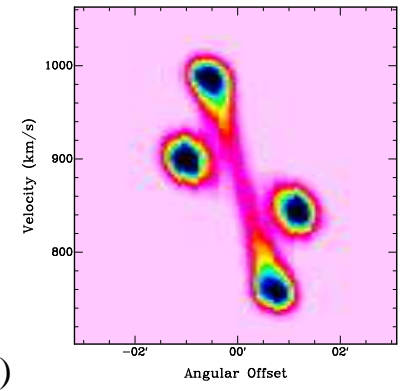

c)



Figure 3: Toy-tilted-ring models of NGC 2685 illustrating that the "Spindle" galaxy cannot have two separate HI rings. a)-c): "Flip"-toymodel, the disk is modelled by two flipped rings. a) Total modelled HI intensity overlaid with the projected rings, b) Parameters of the Flip-model c) Position-velocity-diagram taken along the solid line in Fig.2d). d)-f): "Warp"-toymodel, the disk is modelled as a smoothly twisting structure. d) Total modelled HI intensity overlaid with the projected rings, e) Parameters of the Warp-model f) Position-velocity-diagram taken along the solid line in Fig.2d). The Warp-toymodel reproduces the observed features in the HI total intensity map, as well as in the position-velocity diagram taken from the observations (Figs. 2a) and 2f)). The Flip-model fails to reprduce these. 